\title{
Malnutrition prevalence among children and women of reproductive age in Mexico by wealth, education level, urban/rural area and indigenous ethnicity
}

\author{
Carolina Batis ${ }^{1} \odot$, Edgar Denova-Gutiérrez ${ }^{2}$, Bárbara I Estrada-Velasco ${ }^{3}$ and \\ Juan Rivera ${ }^{2, *}$ \\ ${ }^{1}$ CONACYT - Health and Nutrition Research Center, National Institute of Public Health, Cuernavaca 62100 , \\ Morelos, Mexico: ${ }^{2}$ Health and Nutrition Research Center, National Institute of Public Health, Cuernavaca 62100, \\ Morelos, Mexico: ${ }^{3}$ División de Ciencias de la Salud, Universidad Anáhuac Querétaro, Querétaro 76246, \\ Querétaro, Mexico
}

Submitted 23 January 2019: Final revision received 7 0ctober 2019: Accepted 7 November 2019: First published online 9 March 2020

\begin{abstract}
Objective: To compare the prevalence of malnutrition (undernutrition and excess weight) by wealth, education level, ethnicity and urban/rural areas in Mexican children and women of reproductive age.

Design: We compared the prevalence of overweight, obesity, wasting/underweight, stunting/short stature and anaemia by socioeconomic and ethnic indicators. For each indicator, we estimated prevalence ratios (PR) adjusted by all other socioeconomic and ethnic indicators. We analysed if results differed by urban/rural areas. Setting: Mexican National Health and Nutrition Survey 2012.

Participants: Children <5years, non-pregnant women 11-19years and nonpregnant women 20-49 years ( $n 33244$ ).

Results: In most age groups, belonging to non-indigenous households, with high wealth, high education and in urban areas were inversely associated with stunting or short stature (PR ranging from 0.40 to 0.83 ), and wealth and education were inversely associated with anaemia (PR ranging from 0.53 to 0.78 ). The prevalence of overweight was similar across subgroups among children $<5$ years; however, among women 11-19 years, wealth, non-indigenous household and urban areas were positively associated (PR ranging from 1.16 to 1.33 ); and among women 20-49 years, education was inversely associated (PR 0.83).

Conclusions: Socially disadvantaged populations have a higher prevalence of undernutrition, whereas the prevalence of excess weight is either equal (children $<5$ years), slightly lower (women 11-19 years) or even higher (women 20-49 years) with lower education. These results highlight the need for specific actions to address social inequalities in malnutrition in the Mexican population.
\end{abstract}

Keywords
Social inequalities in malnutrition
Mexico
Wealth
Education
Ethnicity

Social inequalities in health refer to unfair and unnecessary disparities that systematically burden populations rendered vulnerable by social or political structures ${ }^{(1)}$. Malnutrition is a key contributor to this type of inequality. Undernutrition, for instance, is both a cause and a consequence of poverty ${ }^{(2)}$, and obesity is related to factors such as family income or urban/rural area of residence ${ }^{(3)}$. Therefore, to acknowledge and ultimately decrease social inequalities in health, it is important to understand how various factors, including malnutrition, contribute to these disparities.

Malnutrition refers to all forms of nutrition disorders caused by a complex array of factors, including dietary inadequacy (deficiencies, excesses or imbalances in energy, protein and micronutrients), and consists of both undernutrition and excess weight ${ }^{(4)}$. Undernutrition, from conception to the first 2 years of life, has adverse effects

Disclaimer: This paper was published as part of a Supplement in the Journal of Public Health Nutrition, publication of which was supported partially by SLAN CAPÍTULO MEXICO A.C. The papers included in this supplement were invited by the Guest Editor and have undergone the standard journal formal review process. They may be cited. 
on growth, mental and capacity development and intellectual performance ${ }^{(5)}$. Anaemia is partly linked to nutritional deficiencies, but also other causes such as infectious diseases and genetic disorders - the relative importance of each of these factors depending on the context ${ }^{(4,6)}$. It has serious consequences on the motor and intellectual development of children, and, if not corrected before 2 years of age, this damage is irreversible. In women of reproductive age, anaemia is associated with increased maternal and perinatal mortality, increased prematurity and low-birth-weight products $^{(7)}$. On the other hand, overweight and obesity in childhood can have a significant impact on physical and psychological health. Obese and overweight children are likely to stay obese into adulthood and more likely to develop diabetes and cardiovascular diseases at a younger age, and are at a higher risk of breast, colon, prostate, endometrium, kidney and gallbladder cancer ${ }^{(8)}$.

In Mexico, excess weight has become a public health concern; in 2012, $10 \%$ of preschool-aged children, $~ 35 \%$ of school-aged children and adolescents and $\sim 70 \%$ of adults were overweight (including obesity) ${ }^{(9,10)}$. Although undernutrition has declined, it still affects an important part of the population. Around 1.5 million Mexican children $<5$ years $(13.6 \%)$ suffer from stunting (low heightfor-age $)^{(11)}$. In 2012, the prevalence of anaemia among children $<5$ years was $23.3 \%^{(12)}$, and among nonpregnant women aged $12-49$ years, it was $11.6 \%{ }^{(13)}$.

Previous studies looking at malnutrition differences by socioeconomic or ethnic indicators in Mexico have focused on only condition (either overweight/obesity, stunting or anaemia); on only one specific age group; and the majority on only one socioeconomic or ethnicity indica$\operatorname{tor}^{(9-14)}$. Therefore, to better understand social inequalities in all forms of malnutrition, our aim was to estimate parallel to each other and do a comparative analysis of the prevalences of both undernutrition (wasting/underweight, stunting/short stature and anaemia) and excess weight (overweight and obesity); in different age groups (children $<5$ years, women 11-19years, and women 20-49 years); and by several socioeconomic and ethnicity indicators (wealth based on household characteristics and goods, education level and indigenous status). This study is part of a supplemental issue with the participation of several Latin American countries. It was coordinated by young researchers who participated in the VII Latin American Workshop on Leadership in Nutrition (Programa LILANUT) organised by the Latin American Society of Nutrition (SLAN) ${ }^{(15)}$. The goal was to perform analyses that were as comparable as possible across countries. Decisions about the studied population, malnutrition and socioeconomic and ethnic indicators used, and participating countries. Besides the common analyses, in this study we present malnutrition prevalence by urban/rural area, estimate adjusted prevalence ratios (PR) to understand the independent effect of each factor, and examine if the associations were different in rural $v$. urban areas.

\section{Methods}

\section{Participants}

The Mexican National Health and Nutrition Survey 2012 (ENSANUT, its acronym in Spanish) is a cross-sectional, multi-stage probabilistic survey representative of the national, regional and rural/urban population ${ }^{(16)}$. It was carried out between October 2011 and May 2012. The general aim of the survey was to quantify the distribution of health and nutrition status among the Mexican population and monitor trends across time. We included children $<5$ years old ( $n 10658$ ) and non-pregnant women of reproductive age (11-19 years: $n$ 8044; 20-49 years: $n$ 14542).

\section{Malnutrition indicators}

Trained personnel measured weight and height (or length among <2-year-olds) using standardised protocols and equipment. As for indicators of malnutrition, we included overweight and obesity (excess weight), wasting/ underweight, stunting/short stature and anaemia (undernutrition). We estimated BMI as weight ( $\mathrm{kg}$ ) divided by height $(\mathrm{m})$ squared $\left(\mathrm{kg} / \mathrm{m}^{2}\right)$. Definitions were based on the WHO (see Tables' footnote for specific cut-points in the Results section) ${ }^{(17-19)}$. The definition of overweight includes obesity (e.g., overweight is defined as BMI $\geq 25.0 \mathrm{~kg} / \mathrm{m}^{2}$, not BMI $\geq 25$ and $<30$ ). We excluded values with height-for-age $z$-score $<-6$ or $>6$, weight-for-height $z$-score $<-5$ or $>5$, BMI-for-age $z$-score $<-5$ or $>5$, and among women $20-49$ years - BMI $<10$ or $>58 \mathrm{~kg} / \mathrm{m}^{2}$.

$\mathrm{Hb}$ concentration was measured in capillary blood using a portable photometer (HemoCue). Anaemia was defined as $\mathrm{Hb}<110 \mathrm{~g} / \mathrm{l}$ for children $<5$ years and $<1201$ for women 11-49 years. Hb was adjusted to altitude with the Cohen and Haas equation ${ }^{(20)}$. We excluded $\mathrm{Hb}$ values that were $<40$ or $>185 \mathrm{~g} / \mathrm{l}$.

\section{Socioeconomic characteristics and ethnicity}

We included wealth, education level, urban/rural residence area and ethnicity (indigenous/non-indigenous) as socioeconomic and ethnic indicators. Wealth categories were based on a well-being condition index previously estimated with principal component analysis, which included household characteristics and assets such as household's material on the floor, walls and roof, availability of public sanitary sewer system, public water network connected to household, electricity, motor vehicle, television, computer and refrigerator. The first component was used for the index; it explained $40.5 \%$ of the total variation $^{(21)}$. Households were classified into tertiles as low, medium and high wealth. We classified education level as low (0-6 years of schooling: primary school or less), medium (7-12 years of schooling: secondary to high school) and high ( $>12$ years of schooling: more than high school). For children and women <20 years, we used 
mother's education level; and for women 20-49 years, we used their own education level. We defined rural areas according to the Mexican Institute of Statistics and Geography (INEGI, its acronym in Spanish) as areas with $<2500$ inhabitants ${ }^{(22)}$. A household was classified, following ENSANUT's methodology, as indigenous if at least one woman $>12$ years spoke an indigenous language ${ }^{(11)}$. This definition is used by the Mexican National Commission for the Development of Indigenous Populations considering that the identification and transmission of culture occurs within a household, and that decisions, resources, territory and social networks are shared within a household ${ }^{(23)}$.

\section{Statistical analysis}

We calculated means or proportions of age, sex (only among children $<5$ years), education level (only among women 20-49 years), ethnicity and household characteristics and goods in the whole sample and by wealth tertiles.

For each malnutrition indicator, we estimated the prevalence by age group in the whole sample and by wealth tertiles, education level categories, ethnicity and urban/rural area. We tested the differences in the prevalence between each pair of subgroups using the test for linear combinations (lincom command). To quantify the association between socioeconomic and ethnic indicators and the prevalence of overweight or obesity, stunting/short stature and anaemia, we performed generalised linear models with a binomial family and log link to estimate PR. We estimated crude PR and adjusted PR by other socioeconomic indicators plus continuous age. We estimated PR instead of prevalence OR because, although both measures have issues ${ }^{(24)}$, PR was more interpretable as it related to the prevalence presented in the initial analyses. To understand if the later analyses differed by urban/rural area, we repeated the same multiple models but adding an interaction term between each sociodemographic and ethnic indicator and urban/rural area (we ran separate models for each sociodemographic variable). We present the predicted prevalence when the interaction term had a $P$-value $<0 \cdot 10$. We performed a 'chunk test' to jointly test the interaction terms in the case of sociodemographic variables with dummies. As a sensitivity analysis, we estimated the adjusted predicted prevalences of malnutrition by wealth quintiles and deciles to find if there were different patterns or inflection points that we were missing by conducting the analysis by tertiles (see online supplementary material, Supplemental Fig. 1). We conducted these analyses in STATA 14 (StataCorp) using the survey prefix command (svy) to account for the complex design of ENSANUT 2012.

\section{Results}

Participants' characteristics (age, sex and education level), as well as ethnicity and household characteristics and goods by wealth tertiles in ENSANUT 2012 are shown in Table 1. We found that some of the larger differences in household characteristics and goods between low and high wealth were public water network connected to household (22.5\% in low wealth $v .95 .1 \%$ in high wealth), motor vehicle $(14.4 v .67 .4 \%)$, computer $(3.2 v .60 .9 \%)$ and telephone $(6.6 v .72 \cdot 3 \%)$ ownership.

The crude prevalence of malnutrition by socioeconomic indicators among age groups in Mexico is presented in Table 2 . In children $<5$ years, the prevalence of undernutrition (wasting, stunting and anaemia) decreased with increasing wealth and education, while the prevalence of wasting and stunting was higher among indigenous and rural children. Additionally, the prevalence of obesity increased slightly with increasing wealth (1.0, 2.9 and $2.5 \%$ for low, medium and high wealth, respectively) and was higher in urban than rural residents $(2 \cdot 3 \mathrm{v}$. $1.5 \%)$. In women 11-19 years, the prevalence of stunting was higher among all socially disadvantaged groups (low wealth tertile, mothers with low education level, indigenous and rural areas) compared with their counterparts. The prevalence of anaemia decreased with increasing wealth, and there were no differences in the prevalence of underweight. The prevalence of overweight and obesity increased with increasing wealth (high $v$. low wealth: $39 \cdot 0$ v. $29.9 \%$ for overweight; $13.9 v .8 .3 \%$ for obesity), education and among non-indigenous and urban women. In women 20-49 years, short stature was higher among all socially disadvantaged groups; anaemia was higher among the low wealth group and in urban population, whereas underweight was higher among women with medium and high education level. The results for excess weight varied by socioeconomic and ethnicity indicators. For wealth, the prevalence of overweight was slightly higher among medium wealth than low wealth tertile (72.7 v. 69.2\%). However, by education level, the prevalence of overweight and obesity was much lower for women with a higher education level (e.g., obesity prevalence was $41.5 \%$ for low, $34.9 \%$ for medium and $25.9 \%$ for high education level). Whereas obesity was higher among non-indigenous and rural populations.

In Table 3 we present the crude and adjusted prevalence of wasting/underweight, stunting/short stature, anaemia and overweight. As can be seen, adjusted prevalences were weaker than crude prevalences, but adjusted prevalences remained statistically significant in most cases. Moreover, we found that, in general, the associations were much stronger for stunting/short stature and anaemia than for overweight.

In Fig. 1, we present adjusted prevalences by socioeconomic and ethnic indicators in rural and urban areas. We present only the cases in which the interaction between urban/rural areas and the socioeconomic or ethnic indicator was statistically significant $(P<0 \cdot 10)$. For overweight among children and women 11-19 years in rural areas, those with high wealth or education had a higher 
Table 1 Sample characteristics by tertiles of wealth in Mexico - Mexican National Health and Nutrition Survey (ENSANUT 2012)

\begin{tabular}{|c|c|c|c|c|c|c|c|c|c|c|c|c|}
\hline & & & & & & & & Wealth & & & & \\
\hline & \multicolumn{3}{|c|}{ All } & \multicolumn{3}{|c|}{ Low tertile } & \multicolumn{3}{|c|}{ Medium tertile } & \multicolumn{3}{|c|}{ High tertile } \\
\hline & $\%$ & & $95 \% \mathrm{Cl}$ & $\%$ & & $95 \% \mathrm{Cl}$ & $\%$ & & $95 \% \mathrm{Cl}$ & $\%$ & & $95 \% \mathrm{Cl}$ \\
\hline \multicolumn{13}{|l|}{ Children $<5$ years $(n 10658)$} \\
\hline \multicolumn{13}{|l|}{ Age (years) } \\
\hline$n$ & & $2 \cdot 6$ & & & 2.5 & & & $2 \cdot 6$ & & & $2 \cdot 6$ & \\
\hline $95 \% \mathrm{Cl}$ & & $2 \cdot 5,2 \cdot 6$ & & & $2 \cdot 5,2 \cdot 6$ & & & $2 \cdot 5,2 \cdot 7$ & & & $2 \cdot 5,2 \cdot 7$ & \\
\hline Male sex & $50 \cdot 2$ & & $48 \cdot 6,51 \cdot 9$ & $51 \cdot 8$ & & $49 \cdot 6,54 \cdot 1$ & $49 \cdot 7$ & & $47 \cdot 2,52 \cdot 2$ & $49 \cdot 2$ & & $45 \cdot 7,52 \cdot 7$ \\
\hline \multirow{2}{*}{\multicolumn{13}{|c|}{$\begin{array}{l}\text { Women } 11-19 \text { years }(n 8044) \\
\text { Age (years) }\end{array}$}} \\
\hline & & & & & & & & & \multicolumn{4}{|c|}{ Age (years) } \\
\hline$n$ & & $15 \cdot 4$ & & & $15 \cdot 2$ & & & $15 \cdot 4$ & & & $15 \cdot 5$ & \\
\hline $95 \% \mathrm{Cl}$ & & $15 \cdot 3,15 \cdot 5$ & & & $15 \cdot 1,15 \cdot 4$ & & & $15 \cdot 2,15 \cdot 6$ & & & $15 \cdot 4,15 \cdot 7$ & \\
\hline \multicolumn{13}{|l|}{ Women 20-49 years ( $n$ 14 542) } \\
\hline \multicolumn{13}{|l|}{ Age (years) } \\
\hline$n$ & & 33.7 & & & 32.9 & & & 33.6 & & & $34 \cdot 3$ & \\
\hline $95 \% \mathrm{Cl}$ & & $33.5,33.9$ & & & $32 \cdot 6,33 \cdot 2$ & & & $33 \cdot 2,33.9$ & & & $33.9,34.7$ & \\
\hline \multicolumn{13}{|l|}{ Education level } \\
\hline Low (0-6 years) & $30 \cdot 1$ & & $28 \cdot 8,31 \cdot 4$ & 53.0 & & $50 \cdot 6,55 \cdot 3$ & $32 \cdot 7$ & & $30 \cdot 6,34.8$ & $14 \cdot 2$ & & $12 \cdot 7,15 \cdot 8$ \\
\hline Medium (7-12 years) & 51.9 & & $50 \cdot 5,53 \cdot 3$ & $43 \cdot 6$ & & $41 \cdot 4,45 \cdot 8$ & $56 \cdot 6$ & & $54 \cdot 4,58 \cdot 8$ & $53 \cdot 2$ & & $50 \cdot 9,55.5$ \\
\hline High (>12 years) & $18 \cdot 0$ & & $16 \cdot 8,19 \cdot 2$ & 3.5 & & $2 \cdot 8,4 \cdot 3$ & $10 \cdot 7$ & & $9 \cdot 4,12 \cdot 2$ & $32 \cdot 6$ & & $30.4,34.9$ \\
\hline \multicolumn{13}{|l|}{ Households ( $n 23338$ ) } \\
\hline \multicolumn{13}{|l|}{ Ethnicity } \\
\hline Indigenous & $7 \cdot 5$ & & $6 \cdot 6,8 \cdot 5$ & $18 \cdot 3$ & & $15 \cdot 9,21 \cdot 1$ & $6 \cdot 3$ & & $5 \cdot 2,7 \cdot 5$ & $2 \cdot 0$ & & $1.5,2.6$ \\
\hline Non-indigenous & $92 \cdot 5$ & & $91 \cdot 5,93 \cdot 4$ & $81 \cdot 7$ & & $78 \cdot 9,84 \cdot 1$ & 93.7 & & $92 \cdot 5,94 \cdot 8$ & $98 \cdot 0$ & & $97.4,98.5$ \\
\hline \multicolumn{13}{|l|}{ Household characteristics and goods } \\
\hline Bare-earth floor & $3 \cdot 0$ & & $2 \cdot 6,3 \cdot 5$ & 11.5 & & $10 \cdot 1,13 \cdot 0$ & 0.4 & & $0.2,0.7$ & 0.0 & & $0.0,0.0$ \\
\hline Use of firewood or carbon for cooking & $13 \cdot 3$ & & $12 \cdot 4,14 \cdot 3$ & $40 \cdot 9$ & & $38 \cdot 3,43.5$ & 8.4 & & $7 \cdot 3,9 \cdot 6$ & 0.6 & & $0.4,1.0$ \\
\hline Public sanitary sewer system & 77.4 & & $75 \cdot 8,78.9$ & $46 \cdot 8$ & & $43 \cdot 5,50 \cdot 1$ & 79.6 & & $77.5,81 \cdot 6$ & 94.0 & & $93 \cdot 0,95 \cdot 0$ \\
\hline Public water network connected to household & 67.8 & & $66 \cdot 1,69 \cdot 4$ & 22.5 & & $20 \cdot 3,24.9$ & $67 \cdot 7$ & & $65 \cdot 4,70 \cdot 0$ & $95 \cdot 1$ & & $94.0,96 \cdot 0$ \\
\hline Electricity & $99 \cdot 2$ & & $99 \cdot 0,99 \cdot 4$ & 97.5 & & $96 \cdot 7,98 \cdot 2$ & $99 \cdot 6$ & & $99 \cdot 2,99 \cdot 8$ & $99 \cdot 8$ & & $99 \cdot 4,100$ \\
\hline Motor vehicle (car, van, truck or motorcycle) & $42 \cdot 3$ & & $40 \cdot 7,43 \cdot 8$ & 14.4 & & $13 \cdot 0,16 \cdot 0$ & 31.9 & & $29 \cdot 9,33.9$ & 67.4 & & $65 \cdot 0,69 \cdot 6$ \\
\hline Television & $95 \cdot 8$ & & $95 \cdot 3,96 \cdot 2$ & $87 \cdot 1$ & & $85 \cdot 5,88 \cdot 6$ & 97.9 & & $97 \cdot 3,98 \cdot 4$ & 99.2 & & $98.8,99.5$ \\
\hline Computer (laptop or desktop) & $31 \cdot 3$ & & $29 \cdot 8,32 \cdot 8$ & 3.2 & & $2 \cdot 4,4 \cdot 1$ & $15 \cdot 5$ & & $13 \cdot 9,17 \cdot 3$ & $60 \cdot 9$ & & $58 \cdot 6,63 \cdot 2$ \\
\hline Telephone & $40 \cdot 5$ & & $39 \cdot 0,42 \cdot 1$ & $6 \cdot 6$ & & $5 \cdot 5,7 \cdot 8$ & $26 \cdot 4$ & & $24 \cdot 4,28.5$ & $72 \cdot 3$ & & $70 \cdot 3,74 \cdot 3$ \\
\hline Cellphone & 74.6 & & $73.3,75 \cdot 9$ & $52 \cdot 4$ & & $50 \cdot 0,54.9$ & $72 \cdot 8$ & & $70.5,74.9$ & 89.5 & & $87 \cdot 8,90 \cdot 9$ \\
\hline Refrigerator & $87 \cdot 0$ & & $86 \cdot 0,88 \cdot 0$ & $60 \cdot 4$ & & $57 \cdot 9,62 \cdot 9$ & $91 \cdot 6$ & & $90 \cdot 3,92 \cdot 7$ & 99.5 & & $99 \cdot 1,99 \cdot 7$ \\
\hline
\end{tabular}


Table 2 Malnutrition prevalence by wealth, education level, ethnicity and residence area by age groups in Mexico - Mexican National Health and Nutrition Survey (ENSANUT 2012)

\begin{tabular}{|c|c|c|c|c|c|c|c|c|c|c|c|c|}
\hline & \multirow{2}{*}{\multicolumn{2}{|c|}{ Total }} & \multicolumn{6}{|c|}{ Undernutrition } & \multicolumn{4}{|c|}{ Excess weight } \\
\hline & & & \multicolumn{2}{|c|}{$\begin{array}{c}\text { Wasting/ } \\
\text { underweight§ }\end{array}$} & \multicolumn{2}{|c|}{$\begin{array}{l}\text { Stunting/short } \\
\text { staturell }\end{array}$} & \multicolumn{2}{|c|}{ Anemiađ } & \multicolumn{2}{|c|}{$\begin{array}{l}\text { Overweight } \\
\text { (including }^{\text {obesity) }}{ }^{\star \star}\end{array}$} & \multicolumn{2}{|c|}{ Obesity†† } \\
\hline & $n$ & $\%$ & $\%$ & $95 \% \mathrm{Cl}$ & $\%$ & $95 \% \mathrm{Cl}$ & $\%$ & $95 \% \mathrm{Cl}$ & $\%$ & $95 \% \mathrm{Cl}$ & $\%$ & $95 \% \mathrm{Cl}$ \\
\hline \multicolumn{13}{|l|}{ Children $<5$ years } \\
\hline $\begin{array}{l}\text { All } \\
\text { Wealth }\end{array}$ & 10658 & $100 \cdot 0$ & $2 \cdot 8$ & $2 \cdot 4,3 \cdot 3$ & $13 \cdot 6$ & $12 \cdot 6,14 \cdot 7$ & $23 \cdot 3$ & $21 \cdot 7,24 \cdot 9$ & $9 \cdot 7$ & $8 \cdot 8,10 \cdot 6$ & $2 \cdot 1$ & $1 \cdot 8,2 \cdot 6$ \\
\hline Low tertile & 4292 & 33.4 & $5 \cdot 0$ & $4 \cdot 2,6 \cdot 0$ & $22 \cdot 2$ & $20 \cdot 1,24 \cdot 4$ & $26 \cdot 1$ & $23 \cdot 7,28 \cdot 6$ & $8 \cdot 7$ & $7 \cdot 3,10 \cdot 2$ & 1.0 & $0.7,1.4$ \\
\hline Medium tertile & 3717 & 34.5 & $1.6^{*}$ & $1 \cdot 1,2 \cdot 3$ & $10 \cdot 5^{*}$ & $9 \cdot 1,12 \cdot 2$ & 23.0 & $20 \cdot 5,25 \cdot 6$ & 10.4 & $8.9,11.8$ & $2 \cdot 9^{*}$ & $2 \cdot 1,4.0$ \\
\hline High tertile & 2649 & $32 \cdot 1$ & $1 \cdot 8^{*}$ & $1 \cdot 1,2 \cdot 9$ & $8 \cdot 0^{*} \dagger$ & $6 \cdot 6,9 \cdot 8$ & $20 \cdot 4^{\star}$ & $17.4,23.5$ & $10 \cdot 0$ & $8 \cdot 3,11 \cdot 7$ & $2 \cdot 5^{\star}$ & $1.9,3.4$ \\
\hline \multicolumn{13}{|c|}{ Mother’s education levelł } \\
\hline Low (0-6 years) & 3153 & $26 \cdot 6$ & 4.4 & $3.5,5 \cdot 6$ & $21 \cdot 2$ & $18 \cdot 8,23 \cdot 9$ & $24 \cdot 2$ & $21 \cdot 5,26 \cdot 9$ & 8.9 & $7 \cdot 4,10 \cdot 3$ & 1.9 & $1 \cdot 4,2 \cdot 7$ \\
\hline Medium (7-12 years) & 6172 & $60 \cdot 9$ & $2 \cdot 3^{\star}$ & $1 \cdot 8,2 \cdot 8$ & $11 \cdot 6^{\star}$ & $10 \cdot 5,12 \cdot 8$ & 23.9 & $21 \cdot 7,26 \cdot 0$ & $9 \cdot 8$ & $8 \cdot 6,11 \cdot 0$ & $2 \cdot 1$ & $1 \cdot 6,2 \cdot 7$ \\
\hline High (>12 years) & 1000 & $12 \cdot 5$ & $2 \cdot 1^{*}$ & $0.9,5 \cdot 0$ & $8 \cdot 0^{*} \dagger$ & $5 \cdot 6,11 \cdot 4$ & $18 \cdot 6^{*} \dagger$ & $14 \cdot 6,22 \cdot 6$ & $10 \cdot 1$ & $7 \cdot 6,12 \cdot 5$ & $2 \cdot 1$ & $1 \cdot 2,3 \cdot 5$ \\
\hline \multicolumn{13}{|l|}{ Ethnicity } \\
\hline Indigenous & 1302 & 8.7 & $7 \cdot 8$ & $6 \cdot 1,9.9$ & 33.5 & $29.4,37.9$ & $24 \cdot 8$ & $21 \cdot 1,28 \cdot 9$ & $9 \cdot 9$ & $7 \cdot 6,12 \cdot 3$ & $3 \cdot 0$ & $1 \cdot 7,5 \cdot 3$ \\
\hline \multirow{2}{*}{\multicolumn{13}{|c|}{ Residence area }} \\
\hline & & & & & & & & & & & & \\
\hline Rural & 4089 & $25 \cdot 5$ & $4 \cdot 6$ & $3 \cdot 7,5 \cdot 5$ & $20 \cdot 9$ & $18 \cdot 8,23 \cdot 1$ & $25 \cdot 1$ & $22 \cdot 7,27 \cdot 6$ & $8 \cdot 2$ & $7 \cdot 0,9 \cdot 5$ & 1.5 & $1 \cdot 1,2 \cdot 0$ \\
\hline Urban & 6569 & 74.5 & $2 \cdot 2^{*}$ & $1 \cdot 7,2 \cdot 7$ & $11 \cdot 1^{*}$ & $9 \cdot 9,12 \cdot 3$ & $22 \cdot 6$ & $20 \cdot 8,24 \cdot 6$ & $10 \cdot 2^{*}$ & $9 \cdot 1,11 \cdot 3$ & $2 \cdot 3^{*}$ & $1 \cdot 8,2 \cdot 9$ \\
\hline \multicolumn{13}{|l|}{ Women $11-19$ years } \\
\hline $\begin{array}{l}\text { All } \\
\text { Wealth }\end{array}$ & 8044 & $100 \cdot 0$ & 1.6 & $1 \cdot 2,2 \cdot 0$ & $13 \cdot 1$ & $12 \cdot 0,14 \cdot 3$ & $7 \cdot 7$ & $6 \cdot 6,8 \cdot 9$ & $36 \cdot 0$ & $34 \cdot 4,37 \cdot 7$ & $12 \cdot 1$ & $11 \cdot 0,13 \cdot 3$ \\
\hline Low tertile & 2786 & $29 \cdot 3$ & 1.6 & $1 \cdot 1,2 \cdot 3$ & $22 \cdot 7$ & $20 \cdot 1,25 \cdot 4$ & $9 \cdot 7$ & $7 \cdot 8,11 \cdot 6$ & $29 \cdot 9$ & $27 \cdot 2,32 \cdot 7$ & $8 \cdot 3$ & $7 \cdot 0,9 \cdot 7$ \\
\hline Medium tertile & 2737 & $32 \cdot 8$ & 1.8 & $1 \cdot 2,2 \cdot 7$ & $12 \cdot 9^{*}$ & $10 \cdot 9,15 \cdot 2$ & $6 \cdot 9^{*}$ & $5.4,8.4$ & $38 \cdot 0^{*}$ & $35 \cdot 0,41 \cdot 0$ & $13 \cdot 5^{\star}$ & $11 \cdot 6,15 \cdot 7$ \\
\hline High tertile & 2521 & 37.8 & $1 \cdot 3$ & $0.8,2 \cdot 0$ & $5 \cdot 9^{*} \dagger$ & $4 \cdot 8,7 \cdot 2$ & $6 \cdot 8^{*}$ & $4.9,8.7$ & $39.0^{*}$ & $36 \cdot 1,42 \cdot 0$ & $13 \cdot 9^{*}$ & $12 \cdot 1,16 \cdot 0$ \\
\hline \multicolumn{13}{|c|}{ Mother’s education levelł } \\
\hline Low (0-6 years) & 3348 & $46 \cdot 3$ & $2 \cdot 0$ & $1 \cdot 4,2 \cdot 8$ & $17 \cdot 5$ & $15 \cdot 6,19 \cdot 7$ & $7 \cdot 3$ & $5 \cdot 7,8 \cdot 9$ & $33 \cdot 0$ & $30.4,35 \cdot 7$ & 10.5 & $8 \cdot 9,12 \cdot 4$ \\
\hline Medium (7-12 years) & 3011 & $44 \cdot 2$ & 1.3 & $0.8,1.9$ & $9 \cdot 3^{*}$ & $7 \cdot 7,11 \cdot 2$ & $6 \cdot 6$ & $5 \cdot 3,7 \cdot 9$ & $38 \cdot 0^{*}$ & $35 \cdot 4,40 \cdot 7$ & $12 \cdot 9$ & $11 \cdot 2,14 \cdot 7$ \\
\hline High (>12 years) & 543 & 9.5 & $2 \cdot 2$ & $1 \cdot 0,4 \cdot 6$ & $4 \cdot 1^{*} \dagger$ & $2 \cdot 3,7 \cdot 4$ & 9.9 & $3.9,15.9$ & 39.5 & $33 \cdot 2,45 \cdot 9$ & $16 \cdot 6^{*}$ & $12 \cdot 1,22 \cdot 3$ \\
\hline \multicolumn{13}{|l|}{ Ethnicity } \\
\hline Indigenous & 995 & $9 \cdot 8$ & 1.7 & $0.7,3.9$ & 38.8 & $33 \cdot 8,44 \cdot 0$ & $7 \cdot 3$ & $5 \cdot 3,9.9$ & $24 \cdot 6$ & $20 \cdot 6,28 \cdot 6$ & $5 \cdot 0$ & $3 \cdot 6,7 \cdot 0$ \\
\hline Non-indigenous & 7049 & $90 \cdot 2$ & 1.5 & $1 \cdot 2,2 \cdot 0$ & $10 \cdot 3^{*}$ & $9 \cdot 3,11 \cdot 5$ & $7 \cdot 7$ & $6 \cdot 6,9 \cdot 1$ & $37 \cdot 3^{*}$ & $35 \cdot 5,39 \cdot 0$ & $12 \cdot 9^{*}$ & $11 \cdot 7,14 \cdot 2$ \\
\hline \multicolumn{13}{|l|}{ Residence area } \\
\hline Rural & 2913 & $25 \cdot 4$ & $1 \cdot 7$ & $1 \cdot 1,2 \cdot 5$ & 21.5 & $19 \cdot 1,24 \cdot 1$ & 7.5 & $6 \cdot 1,9 \cdot 2$ & $27 \cdot 3$ & $25 \cdot 1,29 \cdot 6$ & $7 \cdot 4$ & $6 \cdot 2,8 \cdot 7$ \\
\hline Urban & 5131 & $74 \cdot 6$ & 1.5 & $1 \cdot 1,2 \cdot 0$ & $10 \cdot 3^{*}$ & $9 \cdot 0,11 \cdot 6$ & $7 \cdot 7$ & $6 \cdot 4,9 \cdot 3$ & $39 \cdot 0^{*}$ & $36 \cdot 9,41 \cdot 1$ & $13 \cdot 7^{*}$ & $12 \cdot 3,15 \cdot 3$ \\
\hline \multicolumn{13}{|l|}{ Women $20-49$ years } \\
\hline All & 14542 & $100 \cdot 0$ & 1.5 & $1 \cdot 2,1.9$ & $21 \cdot 8$ & $20 \cdot 7,22 \cdot 9$ & $13 \cdot 0$ & $12 \cdot 2,14 \cdot 0$ & $70 \cdot 6$ & $69 \cdot 3,71 \cdot 9$ & $35 \cdot 3$ & $34 \cdot 0,36 \cdot 5$ \\
\hline Low tertile & 4925 & $25 \cdot 2$ & $1 \cdot 3$ & $1 \cdot 0,1 \cdot 8$ & $37 \cdot 6$ & $35.4,39.9$ & $14 \cdot 8$ & $13 \cdot 3,16 \cdot 3$ & $69 \cdot 2$ & $67 \cdot 2,71 \cdot 1$ & 34.6 & $32 \cdot 6,36 \cdot 7$ \\
\hline Medium tertile & 4997 & 33.1 & $2 \cdot 1$ & $1.4,3.0$ & $21 \cdot 7^{*}$ & $19 \cdot 9,23.5$ & $14 \cdot 0$ & $12 \cdot 2,15 \cdot 7$ & $72 \cdot 7^{\star}$ & $70 \cdot 6,74 \cdot 8$ & $35 \cdot 7$ & $33 \cdot 6,37 \cdot 7$ \\
\hline High tertile & 4620 & $41 \cdot 7$ & $1 \cdot 2$ & $0.8,1.9$ & $12 \cdot 3^{*} \dagger$ & $10 \cdot 9,13 \cdot 9$ & $11 \cdot 3^{\star} \dagger$ & $9 \cdot 8,12 \cdot 7$ & $69 \cdot 7$ & $67.5,71.9$ & 35.4 & $33 \cdot 3,37 \cdot 5$ \\
\hline \multicolumn{13}{|l|}{ Education level } \\
\hline Low (0-6 years) & 5221 & $30 \cdot 1$ & 0.7 & $0.4,1.0$ & $36 \cdot 4$ & $34 \cdot 2,38 \cdot 7$ & $14 \cdot 1$ & $12 \cdot 5,15 \cdot 7$ & 77.8 & $75 \cdot 8,79 \cdot 8$ & 41.5 & $39 \cdot 3,43 \cdot 6$ \\
\hline Medium (7-12 years) & 7294 & 51.9 & $1.9^{*}$ & $1 \cdot 4,2.5$ & $18 \cdot 1^{*}$ & $16 \cdot 8,19 \cdot 5$ & $13 \cdot 1$ & $11 \cdot 7,14 \cdot 4$ & $70 \cdot 5^{\star}$ & $68 \cdot 8,72 \cdot 2$ & $34.9^{\star}$ & $33 \cdot 2,36 \cdot 7$ \\
\hline High ( $>12$ years) & 2027 & $18 \cdot 0$ & $1.9^{*}$ & $1 \cdot 1,3 \cdot 3$ & $8 \cdot 2^{*} \dagger$ & $6 \cdot 7,10 \cdot 1$ & 11.2 & $8 \cdot 8,13 \cdot 7$ & $58 \cdot 6^{*} \dagger$ & $55 \cdot 1,62 \cdot 1$ & $25 \cdot 9^{*} \dagger$ & $23 \cdot 2,28 \cdot 8$ \\
\hline Ethnicity & & & & & & & & & & & & \\
\hline Indigenous & 1578 & $7 \cdot 5$ & 1.6 & $0 \cdot 5,5 \cdot 1$ & $62 \cdot 2$ & $57 \cdot 7,66 \cdot 5$ & $14 \cdot 6$ & $12 \cdot 1,17 \cdot 6$ & 69.5 & $65 \cdot 7,73 \cdot 3$ & $27 \cdot 8$ & $24.4,31.5$ \\
\hline Non-indigenous & 12964 & $92 \cdot 5$ & 1.5 & $1 \cdot 2,1.9$ & $18 \cdot 5^{*}$ & $17 \cdot 5,19 \cdot 6$ & $12 \cdot 9$ & $12 \cdot 0,13 \cdot 9$ & $70 \cdot 7$ & $69 \cdot 3,72 \cdot 0$ & $35.9^{*}$ & $34 \cdot 6,37 \cdot 2$ \\
\hline Residence area & & & & & & & & & & & & \\
\hline Rural & 9599 & $78 \cdot 8$ & $1 \cdot 6$ & $1 \cdot 2,2 \cdot 0$ & $19 \cdot 0$ & $17 \cdot 8,20 \cdot 3$ & $12 \cdot 8$ & $11 \cdot 7,13 \cdot 9$ & $71 \cdot 0$ & $69 \cdot 5,72 \cdot 5$ & $36 \cdot 2$ & $34 \cdot 7,37 \cdot 7$ \\
\hline Urban & 4943 & $21 \cdot 2$ & 1.5 & $1 \cdot 1,2 \cdot 0$ & $32 \cdot 1^{*}$ & $30 \cdot 0,34 \cdot 2$ & $14 \cdot 0^{\star}$ & $12 \cdot 5,15 \cdot 6$ & $69 \cdot 0$ & $66 \cdot 8,71 \cdot 1$ & $31.9^{*}$ & $29.9,34.0$ \\
\hline
\end{tabular}

$\ddagger$ Weighted \%.

\$Wasting: weight-for-height $z$-score $<-2$ for children $<5$ years; underweight: BMI-for-age $z$-score $<-2$ for women $11-19$ years; and BMI $<18.5$ for women $20-49$ years. IIStunting: height-for-age $<-2$ for children $<5$ years; height-for-age $z$-score $<-2$ for women $11-19$ years; and short stature: height $<1.49 \mathrm{~m}$ for women $20-49$ years.

ПAnemia: $\mathrm{Hb}$ adjusted using the Cohen and Haas equation $<110 \mathrm{~g} / \mathrm{l}$ for children $<5$ years; and $<120 \mathrm{~g} / \mathrm{l}$ for women $11-49$ years. The sample size for anaemia was 7366 for children $<5$ years, 6706 for women $11-19$ years and 12073 among women 20-49 years.

${ }^{* *}$ Overweight (including obesity): BMI-for-age $z$-score $>2$ for children $<5$ years; BMI-for-age $z$-score $>1$ for adolescents $15-19$ years; and BMI $\geq 25 \cdot 0 \mathrm{~kg} / \mathrm{m}^{2}$ for women 20-49 years.

††Obesity: BMl-for-age $z$-score $>3$ for children $<5$ years; BMI-for-age $z$-score $>2$ for women $11-19$ years; and BMI $\geq 30.0$ for women $20-49$ years.

$\ddagger \ddagger$ The sample size by mother's education level was 10325 for children $<5$ years and 6902 for women $11-19$ years.

${ }^{\star} P<0.05$ v. low tertile/low education/indigenous.

$\dagger P<0.05 v$. medium tertile/medium education. 


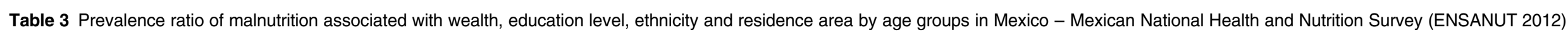

\begin{tabular}{|c|c|c|c|c|c|c|c|c|c|c|c|c|c|c|c|c|}
\hline & \multicolumn{4}{|c|}{ Wasting/undernutrition* } & \multicolumn{4}{|c|}{ Stunting/short stature } & \multicolumn{4}{|c|}{ Anemiał } & \multicolumn{4}{|c|}{ Overweight (including obesity)§ } \\
\hline & \multicolumn{2}{|c|}{ Crude } & \multicolumn{2}{|c|}{ AdjustedII } & \multicolumn{2}{|c|}{ Crude } & \multicolumn{2}{|c|}{ Adjustedll } & \multicolumn{2}{|c|}{ Crude } & \multicolumn{2}{|c|}{ AdjustedII } & \multicolumn{2}{|c|}{ Crude } & \multicolumn{2}{|c|}{ AdjustedII } \\
\hline & $\begin{array}{l}\text { Prevalence } \\
\text { ratio }\end{array}$ & $95 \% \mathrm{Cl}$ & $\begin{array}{l}\text { Prevalence } \\
\text { ratio }\end{array}$ & $95 \% \mathrm{Cl}$ & $\begin{array}{l}\text { Prevalence } \\
\text { ratio }\end{array}$ & $95 \% \mathrm{Cl}$ & $\begin{array}{l}\text { Prevalence } \\
\text { ratio }\end{array}$ & $95 \% \mathrm{Cl}$ & $\begin{array}{l}\text { Prevalence } \\
\text { ratio }\end{array}$ & $95 \% \mathrm{Cl}$ & $\begin{array}{l}\text { Prevalence } \\
\text { ratio }\end{array}$ & $95 \% \mathrm{Cl}$ & $\begin{array}{l}\text { Prevalence } \\
\text { ratio }\end{array}$ & $95 \% \mathrm{Cl}$ & $\begin{array}{l}\text { Prevalence } \\
\text { ratio }\end{array}$ & $95 \% \mathrm{Cl}$ \\
\hline \multirow{2}{*}{\multicolumn{17}{|c|}{ Children $<5$ years }} \\
\hline \multicolumn{5}{|l|}{ Wealth } & & & & & & & & & & & & \\
\hline Low tertile & 1 & & 1 & & 1 & & 1 & & 1 & & 1 & & 1 & & 1 & \\
\hline Medium tertile & $0.31 \pi$ & $0.21,0.47$ & $0.42 ף$ & $0.27,0.66$ & $0.47 \uparrow$ & $0.39,0.579$ & $0.59 \rrbracket$ & $0.49,0.72 \pi$ & 0.88 & $0.76,1.02$ & 0.90 & $0.78,1.05$ & $1 \cdot 19$ & $0.96,1.47$ & $1 \cdot 11$ & $0.88,1.41$ \\
\hline High tertile & $0.36 \pi$ & $0.22,0.61$ & $0.52 \emptyset$ & $0.31,0.85$ & $0.36 \pi$ & $0.29,0.45$ & $0.52 \pi$ & $0.41,0.67$ & $0.78 \pi$ & $0.66,0.93$ & 0.85 & $0.71,1.03$ & $1 \cdot 15$ & $0.90,1.46$ & 1.01 & $0.76,1.35$ \\
\hline Low (0-6 years) & 1 & & 1 & & 1 & & 1 & & 1 & & 1 & & 1 & & 1 & \\
\hline Medium (7-12 years) & $0.52 \eta$ & $0.38,0.71$ & 0.77 & $0.52,1 \cdot 15$ & 0.559 & $0.47,0.64$ & $0.76 ף$ & $0.65,0.89$ & 0.99 & $0.85,1.14$ & 0.98 & $0.85,1.13$ & $1 \cdot 10$ & $0.90,1.35$ & 1.06 & $0.86,1.31$ \\
\hline High ( $>12$ years) & 0.48 & $0.20,1 \cdot 18$ & 0.93 & $0.38,2 \cdot 28$ & $0.38 \pi$ & $0.26,0.55$ & 0.68 & $0.46,1.02$ & 0.779 & $0.60,0.98$ & $0.78 \pi$ & $0.61,0.99$ & 1.14 & $0.85,1.52$ & 1.09 & $0.79,1.51$ \\
\hline \multicolumn{17}{|l|}{ Ethnicity } \\
\hline Indigenous & & & & & & & & & 1 & & 1 & & 1 & & 1 & \\
\hline \multirow{2}{*}{\multicolumn{17}{|c|}{ Residence area }} \\
\hline & & & & & & & & & & & & & & & & \\
\hline Rural & 1 & & 1 & & 1 & & 1 & & 1 & & 1 & & 1 & & 1 & \\
\hline \multirow{3}{*}{\multicolumn{17}{|c|}{$\begin{array}{l}\text { Urban } \\
\text { Women } 11-19 \text { years }\end{array}$}} \\
\hline & & & & & & & & & & & & & & & & \\
\hline Wealth & & & & & & & & & & & & & & & & \\
\hline Low tertile & 1 & & 1 & & 1 & & 1 & & 1 & & 1 & & 1 & & 1 & \\
\hline \multirow{2}{*}{\multicolumn{17}{|c|}{ Mother's education level ${ }^{8}$}} \\
\hline & & & & & & & & & & & & & & & & \\
\hline Low (0-6 years) & 1 & & 1 & & 1 & & 1 & & 1 & & 1 & & 1 & & 1 & \\
\hline Medium (7-12 years) & 0.62 & $0.36,1.06$ & 0.63 & $0.35,1.12$ & $0.53 \rrbracket$ & $0.43,0.66$ & 0.87 & $0.68,1 \cdot 10$ & 0.90 & $0.69,1.18$ & 0.98 & $0.73,1.31$ & $1 \cdot 15 q$ & $1.03,1.28$ & 1.01 & $0.90,1.14$ \\
\hline High ( $>12$ years) & 1.07 & $0.46,2.47$ & 1.32 & $0.52,3.32$ & $0.23 \pi$ & $0.13,0.43$ & 0.519 & $0.27,0.96$ & 1.35 & $0.71,2.56$ & 1.67 & $0.87,3.22$ & 1.20 १ & $1.00,1.43$ & 1.02 & $0.84,1.23$ \\
\hline \multicolumn{17}{|l|}{ Ethnicity } \\
\hline Indigenous & 1 & & 1 & & 1 & & 1 & & 1 & & 1 & & 1 & & 1 & \\
\hline Non-indigenous & 0.93 & $0.38,2.29$ & $1 \cdot 10$ & $0.38,3 \cdot 21$ & $0.27 \rrbracket$ & $0.22,0.32$ & $0.40 \rrbracket$ & $0.34,0.48$ & 1.06 & $0.75,1.49$ & 1.44 & $0.96,2 \cdot 16$ & $1.52 ף$ & $1 \cdot 28,1 \cdot 79$ & $1.32 \emptyset$ & $1.08,1.61$ \\
\hline \multicolumn{17}{|l|}{ Residence area } \\
\hline Rural & 1 & & 1 & & 1 & & 1 & & 1 & & 1 & & 1 & & 1 & \\
\hline Urban & 0.89 & $0.55,1.44$ & 0.98 & $0.52,1.84$ & $0.48 \pi$ & $0.40,0.57$ & 0.90 & $0.73,1.11$ & $0.51 \pi$ & $0.42,0.62$ & 1.16 & $0.86,1.57$ & $1.43 \pi$ & $1.29,1.58$ & $1.33 \pi$ & $1 \cdot 18,1.50$ \\
\hline
\end{tabular}




\section{NS' Public Geath Nurrition}

Table 3 Continued

\begin{tabular}{|c|c|c|c|c|c|c|c|c|c|c|c|c|c|c|c|c|}
\hline & \multicolumn{4}{|c|}{ Wasting/undernutrition* } & \multicolumn{4}{|c|}{ Stunting/short stature $\dagger$} & \multicolumn{4}{|c|}{ Anemiał } & \multicolumn{4}{|c|}{ Overweight (including obesity)§ } \\
\hline & \multicolumn{2}{|c|}{ Crude } & \multicolumn{2}{|c|}{ Adjustedll } & \multicolumn{2}{|c|}{ Crude } & \multicolumn{2}{|c|}{ Adjustedll } & \multicolumn{2}{|c|}{ Crude } & \multicolumn{2}{|c|}{ Adjustedll } & \multicolumn{2}{|c|}{ Crude } & \multicolumn{2}{|c|}{ Adjustedll } \\
\hline & $\begin{array}{l}\text { Prevalence } \\
\text { ratio }\end{array}$ & $95 \% \mathrm{Cl}$ & $\begin{array}{l}\text { Prevalence } \\
\text { ratio }\end{array}$ & $95 \% \mathrm{Cl}$ & $\begin{array}{l}\text { Prevalence } \\
\text { ratio }\end{array}$ & $95 \% \mathrm{Cl}$ & $\begin{array}{l}\text { Prevalence } \\
\text { ratio }\end{array}$ & $95 \% \mathrm{Cl}$ & $\begin{array}{l}\text { Prevalence } \\
\text { ratio }\end{array}$ & $95 \% \mathrm{Cl}$ & $\begin{array}{l}\text { Prevalence } \\
\text { ratio }\end{array}$ & $95 \% \mathrm{Cl}$ & $\begin{array}{l}\text { Prevalence } \\
\text { ratio }\end{array}$ & $95 \% \mathrm{Cl}$ & $\begin{array}{l}\text { Prevalence } \\
\text { ratio }\end{array}$ & $95 \% \mathrm{Cl}$ \\
\hline \multicolumn{17}{|l|}{ Women 20-49 years } \\
\hline $\begin{array}{l}\text { Wealth } \\
\text { Low tertile }\end{array}$ & 1 & & 1 & & 1 & & 1 & & 1 & & 1 & & 1 & & 1 & \\
\hline Medium tertile & 1.55 & $0.96,2.51$ & 1.48 & $0.90,2.44$ & 0.589 & $0.52,0.64$ & 0.759 & $0.68,0.84$ & 0.94 & $0.80,1.11$ & 0.92 & $0.79,1.08$ & 1.059 & $1.01,1.10$ & 1.059 & $1.00,1.09$ \\
\hline High tertile & 0.93 & $0.55,1.56$ & 0.85 & $0.44,1.65$ & $0.33 \pi$ & $0.29,0.38$ & $0.52 \rrbracket$ & $0.45,0.61$ & $0.76 \pi$ & $0.64,0.90$ & $0.72 \rrbracket$ & $0.59,0.87$ & 1.01 & $0.97,1.05$ & 1.02 & $0.98,1.07$ \\
\hline Low (0-6 years) & $\begin{array}{l}1 \\
2.93\end{array}$ & & $\begin{array}{l}1 \\
1.84\end{array}$ & & 1 & & 1 & & 1 & & 1 & & $\begin{array}{l}1 \\
0.919\end{array}$ & & 0.98 & \\
\hline & $\begin{array}{l}2.93 \\
2.97\end{array}$ & $\begin{array}{l}1.81,4.74 \\
1.50,5.85\end{array}$ & $\begin{array}{l}1.84 \\
1.88\end{array}$ & $\begin{array}{l}1.16,2.92 \\
0.91,3.86\end{array}$ & $\begin{array}{l}0.501 \\
0.239\end{array}$ & $\begin{array}{l}0.45,0.55 \\
0.18,0.28\end{array}$ & $\begin{array}{l}0 . / 4 \pi \\
0.44 \pi\end{array}$ & $\begin{array}{l}0.67,0.82 \\
0.34,0.56\end{array}$ & $\begin{array}{l}0.93 \\
0.80\end{array}$ & $\begin{array}{l}0.80,1.08 \\
0.62,1.02\end{array}$ & $\begin{array}{l}1.12 \\
1.08\end{array}$ & $\begin{array}{l}0.95,1.31 \\
0.82,1.42\end{array}$ & $\begin{array}{l}0.9171 \\
0.759\end{array}-30$ & $\begin{array}{l}0.88,0.94 \\
0.71,0.80\end{array}$ & 0.98 & $\begin{array}{l}0.94,1.01 \\
0.78,0.89\end{array}$ \\
\hline \multicolumn{17}{|l|}{ Ethnicity } \\
\hline Indigenous & 1 & & 1 & & 1 & & 1 & & 1 & & 1 & & 1 & & 1 & \\
\hline Non-indigenous & 0.95 & $0.29,3.16$ & 0.88 & $0.24,3 \cdot 27$ & 0.309 & $0.27,0.33$ & $0.45 \rrbracket$ & $0.41,0.50$ & 0.88 & $0.72,1.08$ & 0.95 & $0.77,1 \cdot 18$ & 1.02 & $0.96,1.08$ & 1.02 & $0.96,1.07$ \\
\hline \multirow{2}{*}{\multicolumn{17}{|c|}{$\begin{array}{l}\text { Residence area } \\
\text { Rural }\end{array}$}} \\
\hline & 1 & & 1 & & 1 & & 1 & & 1 & & 1 & & 1 & & 1 & \\
\hline Urban & 0.95 & $0.63,1.44$ & 0.93 & $0.58,1.47$ & 1.68 & $1.53,1.85$ & 0.97 & $0.88,1.07$ & 1.09 & $0.95,1.26$ & 1.00 & $0 \cdot 86,1 \cdot 15$ & 0.97 & $0.94,1.01$ & 0.96 & $0.93,1.00$ \\
\hline
\end{tabular}

*Wasting: weight-for-height $z$-score $<-2$ for children $<5$ years; underweight: BMI-for-age $z$-score $<-2$ for women $11-19$ years; and BMI $<18.5$ for women $20-49$ years.

$\dagger$ Stunting: height-for-age $<-2$ for children $<5$ years; height-for-age $z$-score $<-2$ for women $11-19$ years; and short stature: height $<1.49 \mathrm{~m}$ for women $20-49$ years.

$\ddagger$ Anaemia: $\mathrm{Hb}$ adjusted using the Cohen and Haas equation $<110 \mathrm{~g} / \mathrm{l}$ for children $<5$ years, and $<120 \mathrm{~g} / \mathrm{l}$ for women $11-49$ years.

§Overweight including obesity: BMI-for-age $z$-score $>2$ for children $<5$ years; BMl-for-age $z$-score $>1$ for adolescents $15-19$ years; and BMI $\geq 25.0 \mathrm{~kg} / \mathrm{m}^{2}$ for women $20-49$ years.

IIAdjusted by all other variables in the table and age.

$\mathbb{q} P<0.05$. 
(a)

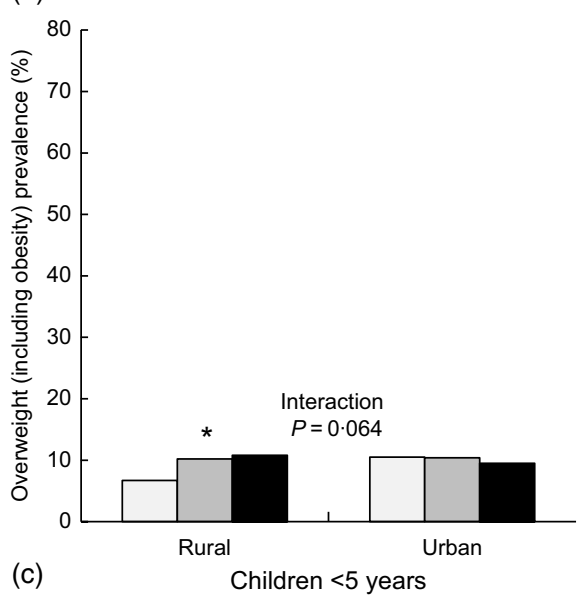

(c)

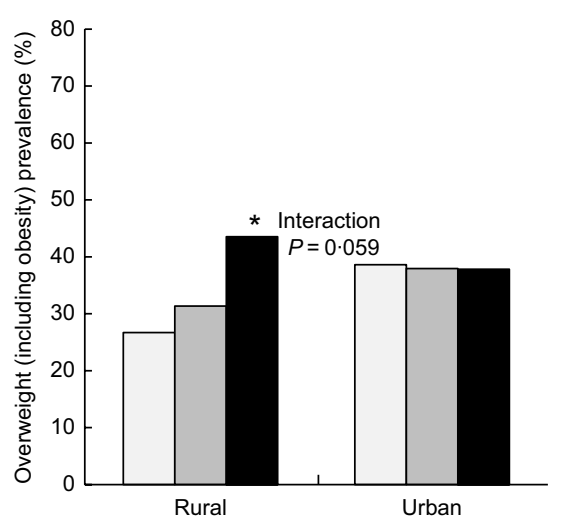

(e)

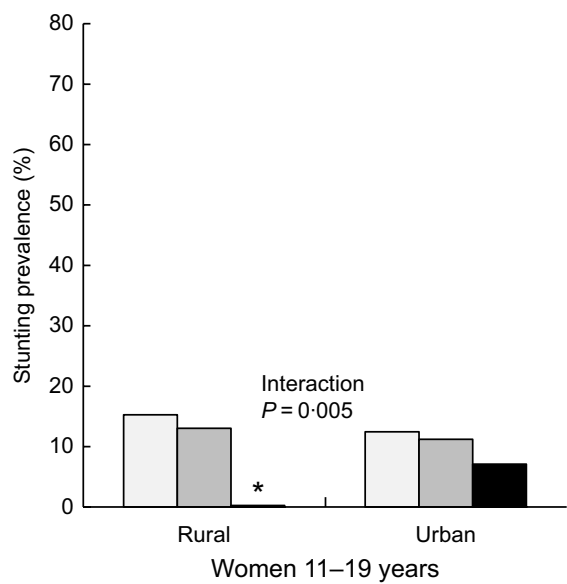

(b)

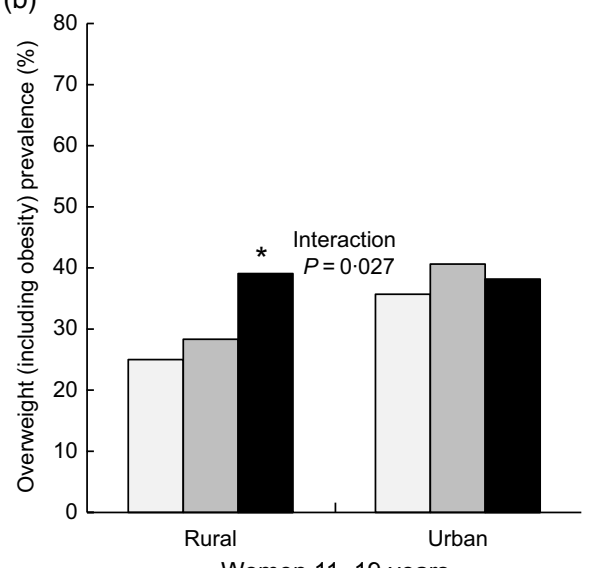

(d)

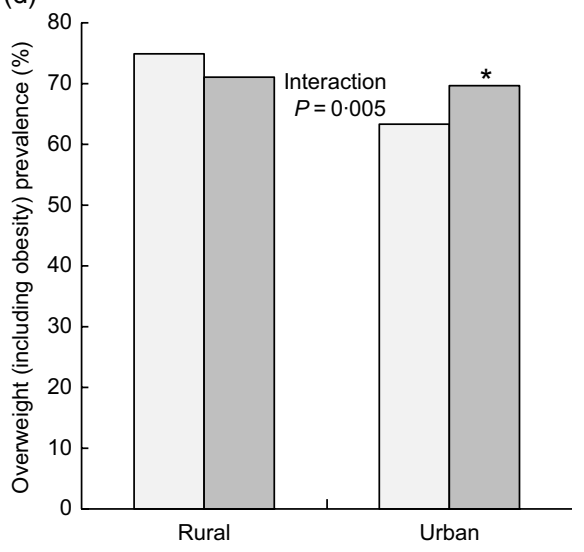

(f)

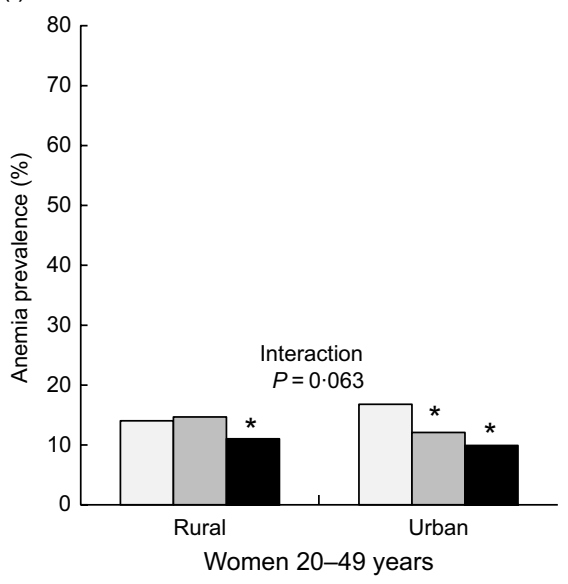

Fig. 1 Predicted prevalence of malnutrition by wealth, education and ethnicity across rural and urban populations. Predictions based on a model that included wealth, education, ethnicity and an interaction term between each of these and urban/rural areas (one model per interaction term). ${ }^{*} P<0.05 \mathrm{v}$. reference category (low wealth, low mother's education or indigenous). Results are only presented for models in which the interaction term had a $P<0.10$. Data are from the Mexican National Health and Nutrition Survey 2012

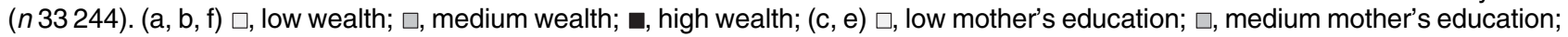
a, high mother's education; (d) $\square$, indigenous; $\square$, non-indigenous

prevalence than those with low wealth or education, whereas in urban areas, there were no differences. Among women 20-49 years, non-indigenous showed a higher prevalence in urban areas, and there were no differences in rural areas. For stunting, the inverse association with education was stronger and statistically significant in rural areas among women 11-19 years; and for anaemia, the inverse association with wealth was stronger in urban areas among women 20-49years. In online supplementary material, Supplemental Fig. 1, we present the adjusted prevalences of malnutrition by wealth tertiles, quintiles and deciles. The shape of the patterns found between malnutrition prevalence as wealth increased remained similar regardless of the level of categorisation of wealth, but the 
gaps between the lowest and highest categories of wealth increased and are more evident when looking at deciles.

\section{Discussion}

In this analysis of a representative sample of the Mexican population, we found important differences in the prevalence of malnutrition by wealth, education, indigenous ethnicity or urban/rural areas. In general, the socially disadvantaged groups (low wealth tertile, low education, indigenous and rural areas) had a higher prevalence of undernutrition, whereas the differences for excess weight were smaller and heterogeneous by age group. For overweight, among children $<5$ years, the prevalence was similar across groups; among women 11-19years, the socially disadvantaged groups had a lower prevalence, and among women 20-49 years, those with low education had a higher prevalence. The strength of associations for these findings was attenuated once we adjusted for all other socioeconomic and ethnic variables, but they remained statistically significant, meaning that each factor had an effect independent of the others. Furthermore, inequality patterns differed by rural and urban populations, among children and adolescents; undernutrition inequalities were larger in the rural population, whereas for overweight in rural population, the socially disadvantaged groups had a lower prevalence, and in urban population, the prevalence was equal.

Regarding our findings for undernutrition, the greatest magnitude in differences between groups was for stunting, caused by a poor diet, insufficient food availability, recurrent infections, limited access to education and health services, which are factors strongly related to poverty $^{(2,25)}$. Moreover, these gaps can persist during generations. The history of undernutrition in parents, especially in the mother, can be determinant since undernutrition during gestation can have adverse effects on the growth and mental development of the child ${ }^{(26)}$. This pattern of stunting being more prevalent among the poor is consistently reported in more than forty low- and middle-income countries ${ }^{(27,28)}$. Strong and tailored social programmes are needed to alleviate this situation. With anaemia, the PR were weaker compared with those observed with stunting, and only statistically significant in the adjusted models for education (among children) and for wealth (among adolescent and adult women). This weaker association with sociodemographic variables observed for anaemia might be related to multiple causes, particularly those that are not modifiable, such as genetic Hb disorders ${ }^{(6)}$.

Contrary to undernutrition, we found that for excess weight, the differences across wealth, education, urban/ rural areas and ethnicity groups were small. In developed countries, obesity is concentrated among the most vulnerable populations, whereas in developing countries, the contrary is the case because those with lower income live in an environment of food scarcity and high energy expenditure $^{(29)}$. According to our results, Mexico is transitioning from one stage to the other. This transition might occur possibly because as the country develops, the obesogenic environment becomes the default in the whole population, including the most vulnerable, and then only individuals with higher income have the resources or motivation to escape that default ${ }^{(30)}$. Another possibility is that the environment itself switches and becomes more obesogenic among the underserved populations (e.g., so-called food-deserts) ${ }^{(31)}$. Furthermore, within Mexico, the transition might be occurring at different rates. Children and adolescents in rural areas still behave as in low-income countries (e.g., those with higher education and wealth had a higher prevalence); in urban areas, they are undergoing the transition (e.g., there were no differences); and among adult women, the trend is following the pattern of high-income countries (e.g., privileged populations tended to have a lower prevalence). The understanding of how this is influenced by the interplay between environmental and individual factors warrants further study. Moreover, among adult women, the different pattern observed for education $v$. wealth in our findings is noteworthy. For wealth, there was an inverted U-shaped pattern, in which women in both extremes of the wealth index had a lower prevalence compared with the mid-wealth. When looking at this association by deciles of wealth, we found that this trend accentuated; women in the lowest decile had a prevalence of $62 \%$; from deciles 2 nd to 9 th, it was $71-75 \%$; and again in the last decile, it was $65 \%$. It is interesting that only the very poor and the very rich had a remarkably lower prevalence. In the case of education, women with higher education had less overweight than those with lower education, even after adjusting for wealth and other socioeconomic indicators. We hypothesise that education might be related to more nutrition knowledge, motivation or awareness, better employment or higher social class. Interestingly, this pattern of inverse association between education and obesity in adult women has been consistently reported in Mexico since $1988^{(32)}$. Moreover, this pattern is different in men, in whom obesity is positively associated with the household's wealth and not associated with education ${ }^{(14)}$.

In Mexico, over 12 million people are indigenous; they represent $10 \%$ of the Mexican population. They live in the most remote areas of the country and are the most vulnerable to changes in the economic, social, political or ecologic environment ${ }^{(23)}$. According to our analysis, stunting was at least three times more prevalent among the indigenous groups compared with the non-indigenous. Yet, the prevalence of overweight was only lower among the indigenous women 11-20 years, but for other age groups, there was no difference. These results are similar to other Latin American countries. In Guatemala, Bolivia, Peru, Brazil and Ecuador, stunting is two to three times higher 
among indigenous $v$. non-indigenous children ${ }^{(33-35)}$. Regarding overweight, in high-income countries such as Australia, Canada and USA, indigenous populations have a higher prevalence compared with non-indigenous ${ }^{(36,37)}$. Whereas in Latin America, as in our results, the pattern is inconsistent. Among children in Bolivia and Guatemala, the prevalence of overweight is slightly higher among indigenous; in Peru it is lower; whereas in Ecuador, it is similar compared with non-indigenous children ${ }^{(34)}$. This scenario is worrisome; it means that the indigenous population is dealing with the double of malnutrition. Their undernutrition problems have not been resolved, and they now face an obesity prevalence comparable to that of the rest of the population. It is important to understand if the environment surrounding the indigenous population is obesogenic as that of other groups, or if the indigenous population is more vulnerable to even lesser obesogenic environments.

Mexico has a long history of implementing programmes and policies aimed at improving the nutritional status of vulnerable groups ${ }^{(38)}$. Chronic undernutrition or stunting has decreased by $50 \%$ from 1988 to 2012, and those with the lowest wealth had the greatest reductions ${ }^{(11)}$. Yet, we found that high prevalences and inequities remain for undernutrition. One possible explanation is that programmes such as 'Prospera' (formerly Progresa and Oportunidades) have led to an increase in the intake of supplements, but they have not achieved improvements in overall dietary patterns. Moreover, given the high prevalence of overweight and obesity in the socially disadvantaged groups that we reported, it is important that nutritional programmes targeting vulnerable populations include obesity prevention components.

Since 2014, the Mexican Government implemented a national strategy to prevent overweight, obesity and diabetes ${ }^{(39)}$. Among several components, the strategy included taxes to sugar-sweetened beverages and non-basic energy-dense foods. The taxes had a differential effect by wealth in urban areas; households with lower wealth reduced the purchases of taxed products the most ${ }^{(40-42)}$. But the prices of taxed products did not increase substantially in rural areas, ${ }^{(43)}$ and hence this policy had a lower effect in rural areas ${ }^{(44)}$. We found that the prevalence of overweight and obesity is still larger in urban areas compared with rural areas among children and adolescent women, but among adult women, there was no difference in the prevalence, or this was higher among rural areas for obesity. It is important to analyse if national policies to prevent obesity are working across socioeconomic and ethnic groups, or if changes on these are warranted. For example, the government cannot control the industry pricing strategy through taxes, but the tax revenue could be used for other programmes to prevent obesity in rural areas.

Strengths of this analysis include the large, nationally representative sample, the comprehensive malnutrition assessment with excess weight and undernutrition indicators, the several socioeconomic and ethnicity indicators, and the different age groups included. Moreover, our analysis was comparable to that of other Latin American countries taking part in this supplemental issue. One of the main limitations is the cross-sectional nature of the survey, which did not enable us to infer causality among socioeconomic and ethnicity indicators and malnutrition. Additionally, we did not analyse men $11-49$ years, subjects $>49$ years old from both sexes, or abdominal adiposity.

In conclusion, these results urge the need to improve the programmes aimed at decreasing undernutrition in the socially disadvantaged populations, and to include obesity prevention components among these. Moreover, how national obesity prevention policies are working across the different socioeconomic strata should be carefully monitored and correction undertaken in case these are leaving a vulnerable stratum out.

\section{Acknowledgements}

Acknowledgements: The authors thank the Latin American Nutrition Leadership Program (Programa LILANUT) for coordinating this supplement, and the National Institute of Public Health for conducting the National Health and Nutrition Survey (ENSANUT) 2012 and for supporting the authors' work on this article. Financial support: DSM Nutritional Products provided funds for the publication of this manuscript. They had no role in the design, analysis or writing of this article. Conflict of interest: There are no conflicts of interest. Authorship: C.B. and J.R. designed the study; C.B. analysed the data; C.B., B.I.E.S. and E.D.G. wrote the article; J.R. made comments to the article. Ethics of buman subject participation: This study was conducted according to the guidelines laid down in the Declaration of Helsinki, and all procedures involving study participants were approved by the Ethics Committee of the Mexican National Institute of Public Health. Informed consent was obtained from each subject or subject's parent/guardian.

\section{Supplementary material}

For supplementary material accompanying this paper visit https://doi.org/10.1017/S1368980019004725.

\section{References}

1. Krieger N (2001) A glossary for social epidemiology. $J$ Epidemiol Community Health 55, 693-700.

2. Heltberg R (2009) Malnutrition, poverty, and economic growth. Health Econ 18, S77-S88. 
3. Braveman P (2009) A health disparities perspective on obesity. Prev Chronic Dis 6, A91.

4. World Health Organization, UNICEF \& USAID (2015) Improving Nutrition Outcomes With Better Water, Sanitation and Hygiene: Practical Solutions for Policies and Programmes. Switzerland: WHO.

5. Victora CG, Adair L, Fall C et al. (2008) Maternal and child undernutrition: consequences for adult health and human capital. Lancet 371, 340-357.

6. Balarajan Y, Ramakrishnan U, Ozaltin E et al. (2011) Anaemia in low-income and middle-income countries. Lancet 378, 2123-2135.

7. Levy A, Fraser D, Katz M et al. (2005) Maternal anemia during pregnancy is an independent risk factor for low birthweight and preterm delivery. Eur J Obstet Gynecol Reprod Biol 122, 182-186.

8. Sahoo K, Sahoo B, Choudhury AK et al. (2015) Childhood obesity: causes and consequences. I Family Med Prim Care 4, 187-192.

9. Hernández-Cordero S, Cuevas-Nasu L, Morán-Ruán MC et al. (2017) Overweight and obesity in Mexican children and adolescents during the last 25 years. Nutr Diabetes $\mathbf{7}$, e247.

10. Barquera S, Campos-Nonato I \& Hernández-Barrera L (2013) Prevalencia de obesidad en adultos mexicanos, 2000-2012 (Prevalence of obesity in Mexican adults 2000-2012). Salud Publica Mex 55, 151-160.

11. Rivera-Dommarco JÁ, Cuevas-Nasu L, González de Cosío T et al. (2013) Desnutrición crónica en México en el último cuarto de siglo: análisis de cuatro encuestas nacionales (Stunting in Mexico in the last quarter century: analysis of four national surveys). Salud Publica Mex 55, 161-169.

12. de la Cruz-Góngora V, Villalpando S, Mundo-Rosas V et al. (2013) Prevalencia de anemia en niños y adolescentes mexicanos: comparativo de tres encuestas nacionales (Prevalence of anemia in Mexican children and adolescents. Results from three national surveys). Salud Publica Mex 55 , 180-189.

13. Shamah-Levy T, Villalpando S, Mundo-Rosas V et al. (2013) Prevalencia de anemia en mujeres mexicanas en edad reproductiva, 1999-2012 (Prevalence of anemia in reproductive-age Mexican women 1999-2012). Salud Publica Mex 55, 190-198.

14. Quezada AD \& Lozada-tequeanes AL (2015) Time trends and sex differences in associations between socioeconomic status indicators and overweight-obesity in Mexico (20062012). BMC Public Health 15, 1244.

15. Barreto P, Batis C, Bortolini G et al. (2018) Proposal and actions to decrease malnutrition in Latin America and the Caribbean. Food Nutr Bull 39, 290-295.

16. Romero-Martínez M, Shamah-Levy T, Franco-Núñez A et al. (2013) Encuesta Nacional de Salud y Nutrición 2012: Diseño y Cobertura (National Health and Nutrition Survey 2012: design and coverage). Salud Publica Mex 55, S332-S340.

17. World Health Organization (1995) Physical Status: The Use and Interpretation of Anthropometry. Report of a WHO Expert Committee. WHO Technical Report Series no. 854. Geneva: WHO.

18. WHO Multicentre Growth Reference Study Group (2006) WHO Child Growth Standards: Length/Height-for-Age, Weight-for-Age, Weight-for-Length, Weight-for-Height and Body Mass Index-for-Age: Methods and Development. Geneva: World Health Organization.

19. de Onis M, Onyango AW, Borghi E et al. (2007) Development of a WHO growth reference for school-aged children and adolescents. Bull World Health Organ 85, 660-667.

20. Cohen JH \& Haas JD (1999) Hemoglobin correction factors for estimating the prevalence of iron deficiency anemia in pregnant women residing at high altitudes in Bolivia. Rev Panam Salud Pública 6, 392-399.

21 Shamah-Levy T, Mundo-Rosas V \& Rivera-Dommarco JA (2014) La magnitud de la inseguridad alimentaria en México: su relación con el estado de nutrición y con factores socioeconómicos (Magnitude of food insecurity in Mexico: its relationship with nutritional status and socioeconomic factors). Salud Publica Mex 56, 79-85.

22. Glossary. Instituto Nacional de Estadística y Geografía (INEGI). http://en.www.inegi.org.mx/app/glosario/default. html?p=rcmorg (accessed July 2019).

23. Comisión Nacional para el Desarrollo de los Pueblos Indígenas (2015) Indicadores socioeconómicos de los pueblos indígenas de México, 2015 (Socioeconomic indicators of indigenous populations in Mexico). https://www.gob. $\mathrm{mx} / \mathrm{cms}$ /uploads/attachment/file/239921/01-presentacionindicadores-socioeconomicos-2015.pdf (accessed January 2019).

24. Pearce N (2004) Effect measures in prevalence studies. Environ Health Perspect 112, 1047-1050.

25. Peña M \& Bacallao J (2002) Malnutrition and poverty. Annu Rev Nutr 22, 241-253.

26. Martorell R \& Zongrone A (2012) Intergenerational influences on child growth and undernutrition. Paediatr Perinat Epidemiol 26, 302-314.

27. Van de Poel E, Hosseinpoor R, Speybroeck N et al. (2008) Socioeconomic inequality in malnutrition in developing countries. Bull World Health Organ 86, 282-291.

28. Ergo A, Gwatkin DR \& Shekar M (2009) What difference do the new WHO Child Growth Standards make for the prevalence and socioeconomic distribution of undernutrition? Food Nutr Bull 30, 3-15.

29. Monteiro CA, Conde WL, Lu B et al. (2004) Obesity and inequities in health in the developing world. Int $J$ Obes Relat Metab Disord 28, 1181-1186.

30. Rivera JA, Barquera S, González-Cossío T et al. (2004) Nutrition transition in Mexico and in other Latin American countries. Nutr Rev 62, S149.

31. Walker RE, Keane CR \& Burke JG (2010) Disparities and access to healthy food in the United States: a review of food deserts literature. Health Place 16, 876-884.

32. Perez Ferrer C, Mcmunn A, Rivera Dommarco JA et al. (2014) Educational inequalities in obesity among Mexican women: time-trends from 1988 to 2012. PLoS One 9, e90195.

33. Ramirez-Zea M, Kroker-Lobos MF, Close-Fernandez R et al. (2014) The double burden of malnutrition in indigenous and nonindigenous Guatemalan populations. Am J Clin Nutr 100, 1644S-1651S.

34. Lutter CK \& Chaparro CM (2008) Malnutrition in Infants and Young Children in Latin America and the Caribbean: Achieving the Millennium Development Goals. Washington, DC: Pan American Health Organization.

35. Horta BL, Santos RV, Welch JR et al. (2013) Nutritional status of indigenous children: findings from the first national survey of indigenous people's health and nutrition in Brazil. Int $J$ Equity Health 12, 23.

36. Wake M, Hardy P, Canterford L et al. (2007) Overweight, obesity and girth of Australian preschoolers: prevalence and socio-economic correlates. Int J Obes 31, 1044-1051.

37. Schell LM \& Gallo MV (2012) Overweight and obesity among North American Indian infants, children, and youth. Am J Hum Biol 24, 302-313.

38. Barquera S, Rivera-Dommarco J \& Gasca-Garcia A (2001) Políticas y programas de alimentación y nutrición en México (Food and nutrition policies and programs in Mexico). Salud Publica Mex 43, 464-477.

39. Secretaría de Salud (2013) Estrategia Nacional para la Prevención y el Control del Sobrepeso, la Obesidad y la 
Diabetes (National Strategy for the Prevention and Control of Overweight, Obesity and Diabetes). Mexico, D.F.: IEPSA.

40. Colchero MA, Popkin BM, Rivera JA et al. (2016) Beverage purchases from stores in Mexico under the excise tax on sugar sweetened beverages: observational study. BMJ 352, h6704.

41. Batis C, Rivera JA, Popkin BM et al. (2016) First-year evaluation of Mexico's tax on nonessential energy-dense foods: an observational study. PLOS Med 13, e1002057.

42. Cochero MA, Rivera-Dommarco J, Popkin BM et al. (2017) In Mexico, evidence of sustained consumer response two years after implementing a sugar-sweetened beverage tax. Health Aff 36, 564-571.

43. Colchero M, Zavala J, Batis C et al. (2017) Cambios en los bebidas y alimentos con impuesto en áreas rurales y semirrurales de México (Changes in prices of taxed sugar-sweetened beverages and nonessential energy dense food in rural and semi-rural areas in Mexico). Salud Publica Mex 59, 137-146.

44. Hernández-F M, Batis C, Rivera JA et al. (2019) Reduction in purchases of energy-dense nutrient-poor foods in Mexico associated with the introduction of a tax in 2014. Prev Med 118, 16-22. 